Commentary: Pulmonary metastasectomy efficacy: A game of clones?

\author{
Harvey I. Pass, MD
}

In this issue of the Journal, Antonoff and colleagues ${ }^{1}$ provide the most comprehensive discussion of muiltiple aspects of the state of the art for pulmonary metastasectomy published to date, which should be a must-read for thoracic surgeons and thoracic surgeons in training alike. Before commenting on this contribution, however, I think it is important to provide this disclosure/disclaimer. I am a general thoracic surgeon, and yes, I am biased in that I believe there absolutely is a role for the resection of pulmonary metastases in selected patients. Although I am certainly not an expert on this subject, I have been associated with some of the leaders in this discipline and have learned much from them. The true experts wrote the phenomenal manuscript that this commentary is meant to enhance-frankly, a really tough job.

But "winter is coming." Although we certainly can make statements like "in general, there is clear demonstration of improvement in prognosis for appropriately selected surgical patients" ${ }^{2}$ and "surgery for pulmonary metastases is a pillar of modern thoracic surgery," ${ }^{3}$ there are very wellrespected and well-meaning "night kings" who stand ready to wipe these statements off the face of thoracic surgery journals. ${ }^{4-6}$ These night kings' holy grail (or maybe "ice blade") is something that is difficult to argue with: the prospective randomized trial. The authors provide a very nice discussion of the updated Pulmonary

\footnotetext{
From the Department of Cardiothoracic Surgery, NYU Langone Health, New York, NY

Disclosures: The author reported no conflicts of interest.

The Journal policy requires editors and reviewers to disclose conflicts of interest and to decline handling or reviewing manuscripts for which they may have a conflict of interest. The editors and reviewers of this article have no conflicts of interest

Received for publication Oct 13, 2020; revisions received Oct 13, 2020; accepted for publication Nov 15, 2020; available ahead of print Nov 23, 2020.

Address for reprints: Harvey I. Pass, MD, Division of General Thoracic Surgery, Department of Cardiothoracic Surgery, NYU Langone Health, 530 First Ave, 9V, New York, NY 10016 (E-mail: Harvey.pass@nyumc.org).

J Thorac Cardiovasc Surg 2022;163:503-10 $0022-5223 / \$ 36.00$

Copyright $(2020$ Published by Elsevier Inc. on behalf of The American Association for Thoracic Surgery

https://doi.org/10.1016/j.jtcvs.2020.11.049
}

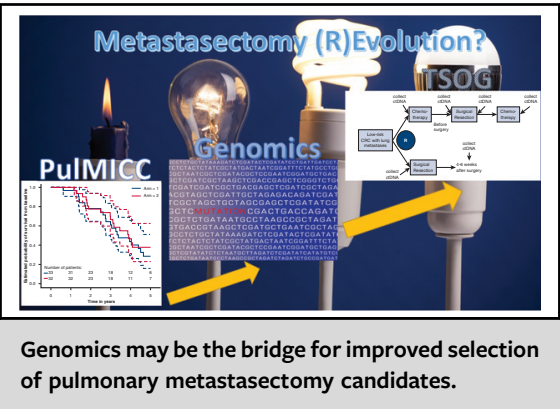

CENTRAL MESSAGE

Pulmonary metastasectomy has

been called into question by a

randomized trial. Prospective tri-

als investigating whether

genomic markers can improve

surgical selection for metasta-

sectomy are needed.

Metastasectomy versus Continued Active Monitoring in Colorectal Cancer (PulMICC) trial, ${ }^{7}$ and thoracic surgeons must be familiar with this trial and its shortcomings. This trial will be quoted mercilessly by medical oncologist (and some thoracic surgeon) "night walkers" to specifically argue against the use of metastasectomy for colorectal pulmonary metastases. Moreover, I worry that there may be an attempt to "generalize" these findings to other histologies that are currently candidates for metastasectomy. Thus, this commentary (which hopefully can be educational) not only summarizes the failings of this trial, which failed to reach accrual, but also aims to convince the thoracic surgical community that selection for metastasectomy remains stuck in the 1990s. The take home point is that leaders in the field, including the authors of this article, are recognizing that not all colorectal metastases are the same even if the disease-free interval is promising, the size is small, or there are not many to deal with. It is time for metastasectomy selection to move into the realm of genomic prognostication in addition to or in place of the usual clinical selection factors.

PulMICC: THE BAD, THE UGLY, BUT SOME GOOD

The PulMICC is the only randomized trial reported to date comparing metastasectomy and nonmetastatic "control data," defined as observation. Eligibility criteria included patients with any number of radiologically presumed or 
biopsied metastases, and the randomization was stratified by age, sex, $\mathrm{T}$ and $\mathrm{N}$ stage of the primary colorectal cancer (CRC), previous hepatic resection, interval from primary resection to randomization, number of metastases, and carcinogenic embryonic antigen level. Patients could not receive any local therapy during the first year after randomization but could have local therapies as indicated thereafter. Chemotherapy could be delivered at any time. This stratification was totally appropriate and reflected many, but not all, the prognostic factors discussed in the Antonoff manuscript. Unfortunately, however, only 93 patients were randomized, and the study called for a 1:1 randomization of 300 patients to show a $10 \%$ difference in overall mortality at 3 years to satisfy noninferiority criteria. The median survival after metastasectomy was 3.5 years $(95 \%$ confidence interval [CI], 3.1-6.6 years), compared with 3.8 years $(95 \%$ CI, 3.1-4.6 years) for controls.

So this trial failed to show a difference between no local option and control, and the authors call for "large definitive randomized trials, investigating the possible benefits of the practice of pulmonary metastasectomy for any tumour type." What could make one think that doing such a trial is going to have any better accrual than PulMICC? And can surgeons really believe that they will be able to stratify patients as being at "higher risk" for local therapy by simply relying on whether they had liver metastases in the past that are now quiescent in addition to clinical prognostic indicators that I used as Jack Roth's fellow at the National Cancer Institute in 1985? Why should anyone interested in this topic think that liver metastases are the only possible harbinger of bad outcomes for patients who would have pulmonary metastasectomy? These patients do not just die from liver metastases, they also die from peritoneal recurrence, brain and bone metastases, local pulmonary complications of endobronchial disease, and obstruction due to lymph node metastases. This is the whole point that only a few people are realizing: "it's the biology, stupid," and unless you are able to predict the biology either of the primary or the metastases in a more objective manner, you will never be able to select the right patient for the right treatment, whether its PulMICC control or "selective" metastasectomy. If the biology is so good in, say, lung cancer so that we can now subselect patients who should or should not receive biological or targeted therapies, then why can we not hypothesize that the biology could also help surgeons (and medical oncologists) to decide whether a patient is a candidate for metastasectomy? Let me make a heretical statement here: maybe not all colon cancers are the same (not so heretical), and maybe there are clinical and molecular aspects of colon cancers that could influence selection of candidates for metastasectomy besides size, disease-free interval, number of metastases, and yes, previous liver metastases. This is the gold nugget in the Antonoff article that needs to be expanded, so let me throw out a few things that I have discovered from the literature (guided by the Antonoff manuscript) that blends the skepticism of PulMICC with what could be explored prospectively to make surgical selection better for metastasectomy candidates.

\section{CRC METAGENOMICS AND PROGNOSTICATION: AS COMPLEX AS ANY TUMOR}

For thoracic surgical oncologists to justify the continued use of metastasectomy in CRC in the face of powerful arguments to the contrary, they must be able to argue that there are insufficient level 1 data to conclude that it does not add benefit in selected cases. Certainly one of the best arguments is the first sentence of the discussion of the PulMICC publication: "Because of recruitment difficulties, PulMiCC closed early, and we were unable to reach the desired statistical endpoints and convincingly answer the question about the value of pulmonary metastasectomy." However, going forward, thoracic surgeons should use every opportunity to equate "selection" with "personalized therapy," as pushed (with good reason) by our medical colleagues, and to enthusiastically "blind (them) with science."

Just like lung cancer, CRC is heterogenous, with genomic and epigenomic influences on prognosis. We have known this since 1993, when Thibodeau and colleagues ${ }^{9}$ reported that microsatellite instability was significantly correlated with the tumor's location, survival, and loss of heterozygosity for chromosomes 5q, 17p, and 18q. In 2002, Vogelstein's group $^{10}$ was the first to actually correlate $K R A S$ and $B R A F$ mutations with CRC, and these oncogenes have maintained a prominent place in CRC biology and prognosis. In 2012, The Cancer Genome Atlas (TCGA) performed an integrative analysis of 224 CRCs that revealed different mutational patterns in the left colon and right colon, frequent mutations of $T P 53$ and $K R A S(60 \%$ and $43 \%$, respectively), as well as a $46 \%$ mutation rate of $B R A F$ in hypermutated tumors. Moreover, an integrated analysis of copy number, gene expression, methylation, and pathway data (Figure 1) defined tumors with greater local or metastatic potential. ${ }^{11}$ More recently, based on the TCGA analysis, CRCs have been more clearly defined by molecular cohorts, which, as shown in Figure 2, have differing overall survival (OS), disease-free survival, and survival after relapse. ${ }^{11}$ Molecular and immune heterogeneity of CRCs has been further illustrated by the finding of 5 immune subtypes of CRC from 572 TCGA CRCs, as shown in Figure $2 .{ }^{12}$ Patients with transforming growth factor $\beta$-dominant and wound healing immune subtypes showed better prognosis (5-year OS of $100 \%$ and $65 \%$, respectively), whereas those with IFN- $\gamma$-dominant and inflammatory immune subtypes were associated with the worst outcomes (5-year OS of $49 \%$ and $23 \%$, respectively). Adding to the phenomenal complexity of CRC heterogeneity and prognostic implications is further evidence that behavior of right-sided versus left-sided CRCs was influenced by variations in their 


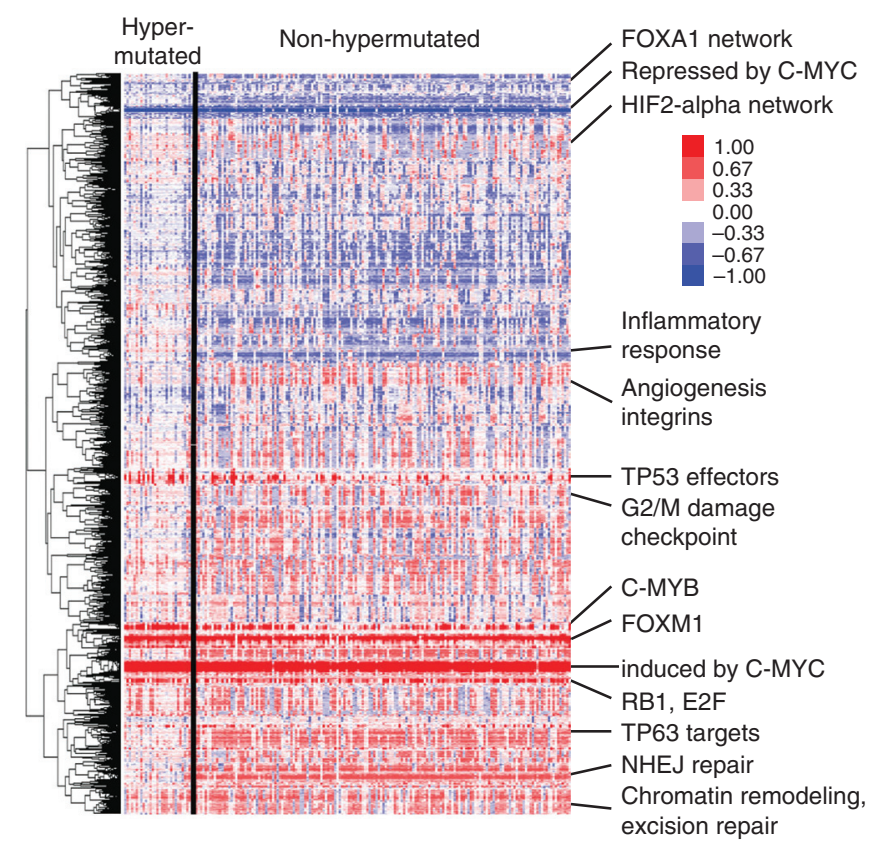

A

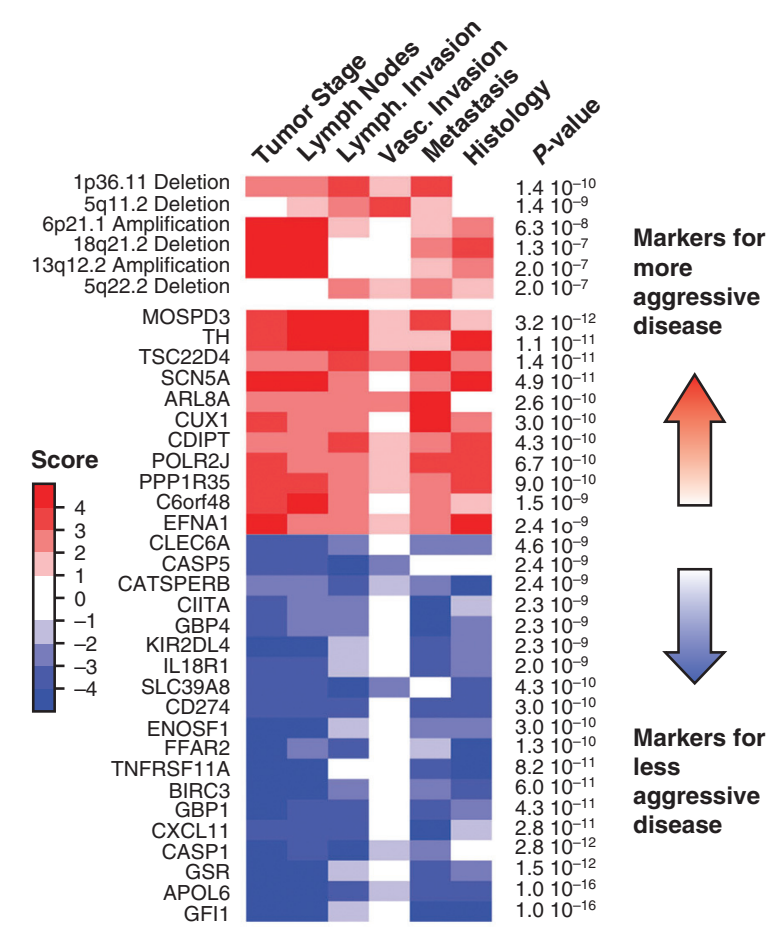

B

FIGURE 1. A, Clustering of genes and pathways affected in colorectal tumors. Blue indicates underexpression relative to normal; red, overexpression relative to normal. B, Gene expression signatures and somatic copy number alterations associated with tumor aggression. Molecular signatures (rows) show significant association with tumor aggressiveness, with red indicating markers of tumor aggressiveness and blue indicating markers of less aggressive tumors. Modified from Cancer Genome Atlas Network. ${ }^{11}$

molecular alterations. ${ }^{13-16}$ In general, right-sided CRCs are enriched for microsatellite- high, hypermethylated $B R A F$ mutant cancers, which may contribute to the poorer prognosis of right-sided CRCs. ${ }^{17}$

\section{SO WHERE DOES PULMONARY METASTASECTOMY FIT WITH THESE FINDINGS?}

In the pre-next-generation sequencing (NGS) era, Schweiger and colleagues ${ }^{19}$ hypothesized in 2014 that "alterations in the EGFR pathway might be relevant in the subset of patients undergoing pulmonary metastasectomy" and used restriction fragment length polymorphism analysis to detect $K R A S$ codons 12 and 13 and BRAF codon 600 in metastases from 39 CRC patients undergoing pulmonary resection. Patients with KRAS mutated tumors had a higher likelihood of developing pulmonary recurrence compared with the KRAS wild-type (WT) group, and KRAS mutation was associated with multiple pulmonary nodules. No $B R A F$ abnormalities were noted, but KRAS mutation analysis was consistent between synchronous pulmonary metastases in the same patient as well as at reoperation (but remember, this is before NGS). In the absence of survival data, this study at least hinted that the risk of subsequent pulmonary recurrence after metastasectomy may be influenced by KRAS status.

This study was then followed by a series of investigations from Renaud and colleagues ${ }^{20-22}$ with a larger, well-defined cohort $(\mathrm{n}=180)$ of CRC metastasectomy patients in which polymerase chain reaction amplification of codon 12/13 KRAS mutations (seen in $52 \%$ of the patients), and V600E for BRAF mutations (seen in $11 \%$ of the patients). WT KRAS tumors differed from mutant KRAS or mutant $B R A F$ tumors by having smaller pT status, an absence of lymph node involvement at the time of metastasectomy, fewer metastases, and fewer previous liver metastases $(P$ $<.02$ for all). Moreover, in multivariate analyses, larger size of the primary tumor, presence of $B R A F$ or KRAS mutation, and Charlson Comorbidity Index were independently associated with risk of death. The absence of $B R A F$ or $K R A S$ mutations along with original tumor $\mathrm{pT}$ status were associated with prolonged recurrence-free survival after metastasectomy. Follow-up data from the Renaud group revealed the importance of VEGF inhibition with bevacizumab in addition to metastasectomy in patients with $K R A S$ mutation. Patient survival after metastasectomy $(P=.001$, bevacizumab vs no bevacizumab; $P=.26$, chemotherapy vs no chemotherapy) and the probability of 


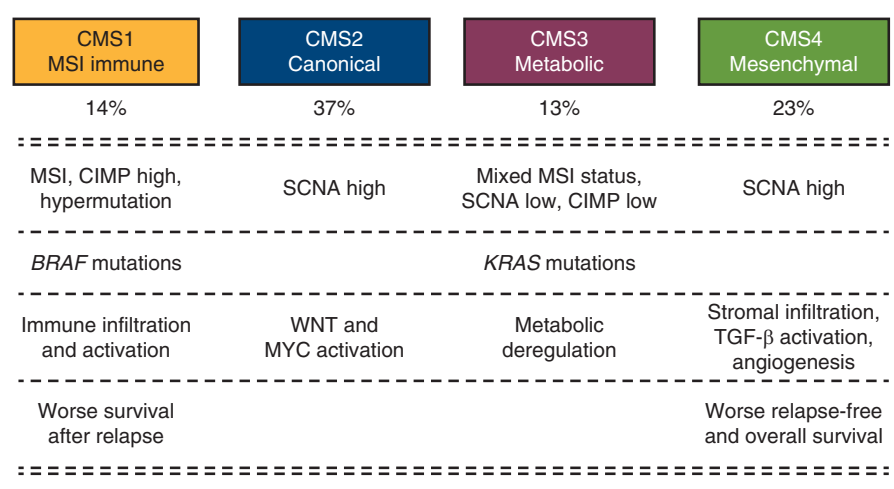

A
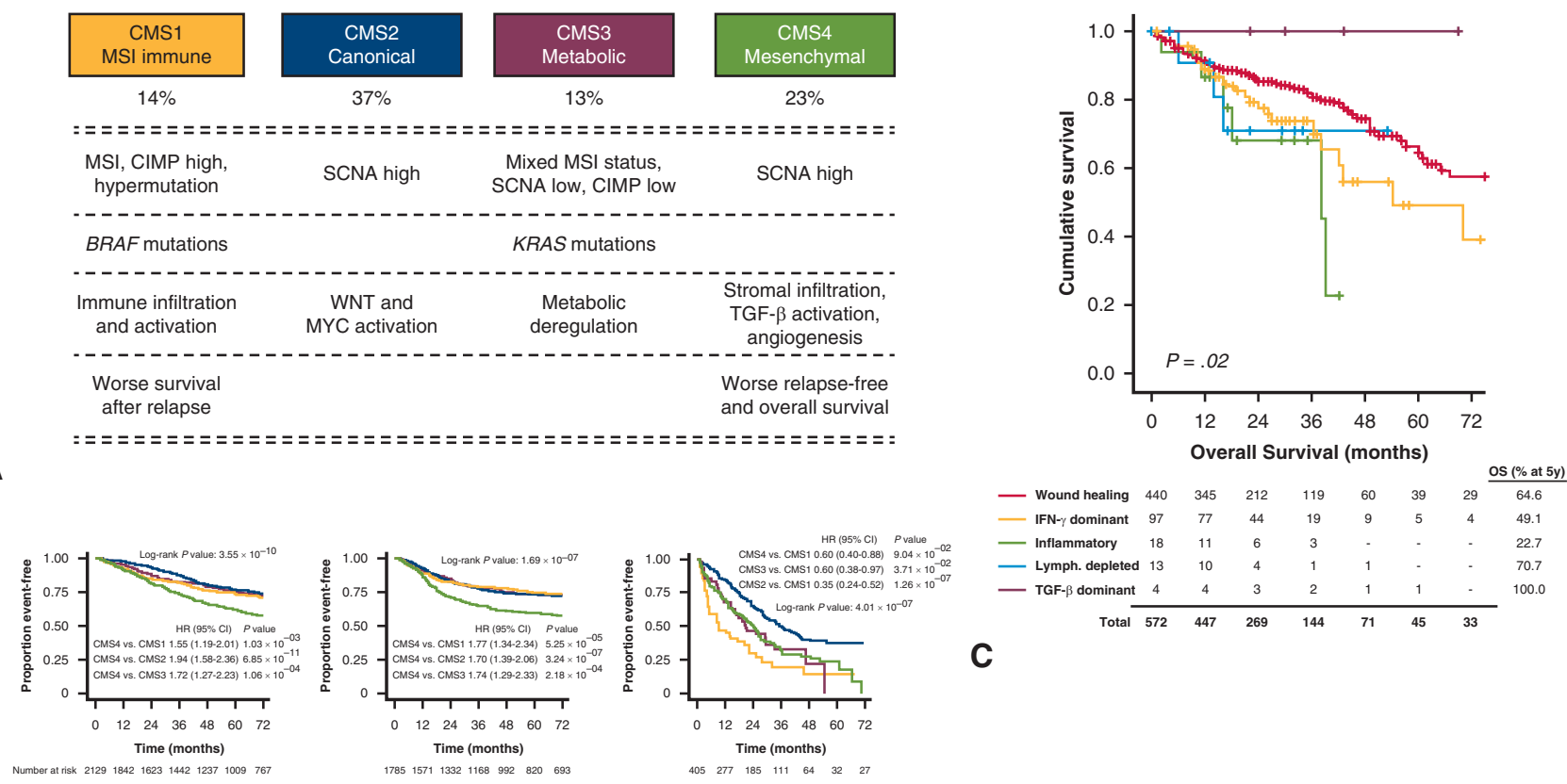

B

FIGURE 2. A, Molecular heterogeneity of colorectal cancer (CRC). Proposed consensus molecular subtypes of CRC, reflecting significant biological differences in the gene expression-based molecular subtypes. CIMP, CpG island methylator phenotype; MSI, microsatellite instability; SCNA, somatic copy number alterations. B, Prognostic associations of consensus molecular subtype groups shown in A. From left to right, Kaplan-Meier survival analysis for overall survival $(n=2129)$, relapse-free survival $(n=1785)$, and survival after relapse $(n=405)$. Modified from Guinney and colleagues. ${ }^{12} \mathrm{C}$, Impact of immune subtypes on survival of CRC patients. Overall survival by immune subtype in The Cancer Genome Atlas CRC cohort $(\mathrm{n}=572)$ reveals differing survival, with patients with transforming growth factor $\beta$-dominant and wound healing immune subtypes living the longest. Modified from Soldevilla and colleagues. $^{13}$

local recurrence after lung metastasectomy $(P=.001$, bevacizumab vs no bevacizumab; $P=.14$, chemotherapy vs no chemotherapy) was influenced more by the use of bevacizumab than by chemotherapy alone in patients with KRAS mutation. This is an important potential stratification demographic for future metastasectomy studies. According to National Comprehensive Cancer Network guidelines, patients eligible for metastasectomy of resectable metachronous lesions are those who have not received previous chemotherapy or who would be treated neoadjuvantly without bevacizumab; however, there is no recommendation for these resected patients to receive adjuvant bevacizumab in the guidelines. ${ }^{23}$

Corsini and colleagues ${ }^{24}$ expanded the number of potential lung metastasectomy prognostic genes beyond $K R A S$ to the RAS family mutations (KRAS, NRAS, and HRAS), as well as to the $A P C$ and TP53 genes in their analyses using Ion Torrent Ampliseq Cancer Panel Primers versions 1.0 and 2.0 for 50 cancer-related genes (Life Technologies, Grand Island, NY). In multivariable analyses, $R A S$, TP53, and $A P C$ mutations were associated with OS in the cohort of 130 patients, whereas disease-free survival was associated with RAS and TP53 mutations. However, the unique quality of this study was that combinations of the genes with or without mutations influenced both cumulative survival and recurrence-free survival. $R A S$ and $A P C$ mutation/WT combinations revealed the best survival with $R A S$ WT and $A P C$ mutation and the worst survival with $R A S$ mutation and $A P C$ WT $(P=.030)$. Longer recurrence-free survival was characteristically seen with TP53 WT and APC mutation.

\section{WHAT GENOMICS ARE IMPORTANT: THE PRIMARY OR THE METASTASES?}

Are there genomic differences between the metastases and the primary CRC? Older studies found high $(85 \%$ $100 \%$ ) oncogene mutational status concordance between the primary tumor and metastases irrespective of patient medical history or treatment duration. ${ }^{25-27}$ Global approaches to describing diversity between the primary tumor and metastases and between metastases have been reported only recently. The report by Schweiger and colleagues $^{29}$ is of greatest interest, because it is one of the few to use the MiSeq platform (Illumina, San Diego, Calif) to describe the mutational profile of lung metastases and their corresponding primary tumors. NGS of CRC pulmonary metastases was successful in 47 patients, and sequencing data were obtained from 24 of the corresponding primary tumors. The usual mutated tumors had rates of $65.5 \%$ and $68.1 \%$ for $A P C, 58.6 \%$ and $57.4 \%$ for 


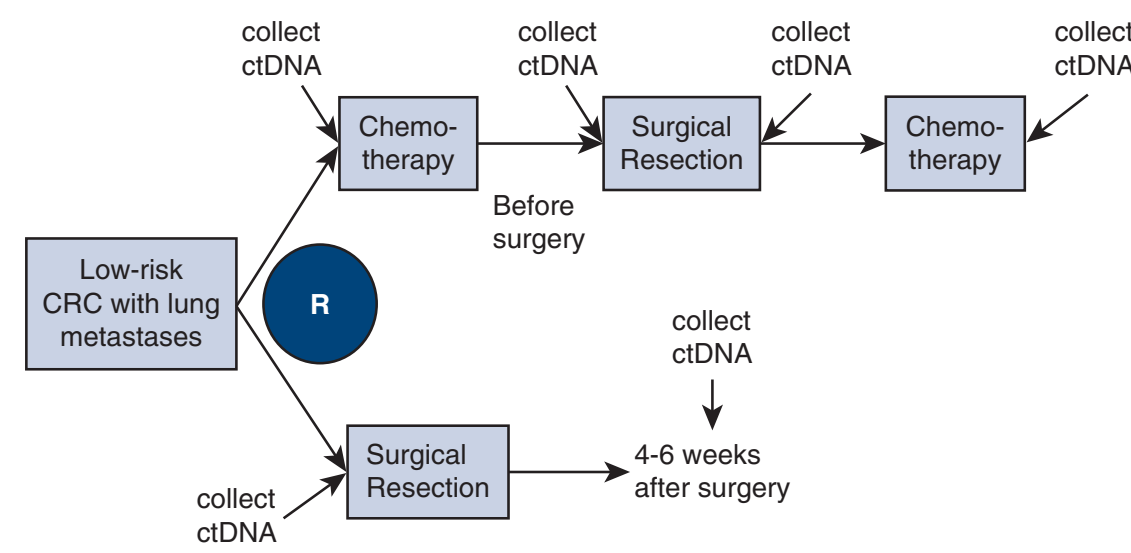

FIGURE 3. The Thoracic Surgery Oncology Group colorectal cancer pulmonary metastasectomy trial incorporates circulating tumor DNA before resection or neoadjuvant chemotherapy, as well as longitudinally, to determine its role as a prognostic or predictive biomarker for patients undergoing complete resection of metastases. $C R C$, Colorectal cancer.

$T P 53$, and $48.3 \%$ and $55.3 \%$ for KRAS in primary tumors and corresponding metastases, respectively. Of interest, mutation frequencies were higher in metastases compared with primary CRCs. In addition, 147 de novo mutations were evident in lung metastases compared with the primary tumors, and 43 mutations were detected in the primary tumors but were absent in the matched metastases. Prognostic modeling using data from NGS revealed that PDGFRA, $T P 53$, and SMARCB1 were associated with OS after pulmonary metastasectomy $(P=.003)$ and $E G F R, G N A Q, K I T$, $M E T$, and PTPN11 were associated with time to recurrence $(P<.001)$. These data hint at a more global approach to prognosticating pulmonary metastases than just choosing a few of the major mutated genes.

\section{COULD METASTASECTOMY RESECTION TECHNIQUE BE INFLUENCED BY GENOMICS?}

Could these $R A S$ mutations potentially influence the thoracic surgeon as to the extent of the metastasectomy for CRC, that is, the type of sublobar resection to perform? Renaud and colleagues ${ }^{30}$ reported a thought-provoking retrospective study limited to wedge resection or segmentectomy for CRC pulmonary metastases with very specific exclusion criteria (only 1 metachronous metastasis, known molecular status, and absence of BRAF mutations). All of the 168 CRC patients $(\mathrm{n}=95$ [57\%] with KRAS mutations) were believed to be cured at the time of the thoracic procedure. The type of resection did not impact the median OS in WT patients $(P=.67)$; however, in multivariate comparisons of anatomic resection compared with wedge resection, the median OS, time to pulmonary recurrence, and margin recurrence rate were significantly better following anatomic resection in patients with KRAS mutations (101 vs 45 months, $P=.02 ; 50$ vs 15 months, $P=.01 ; 4.8 \%$ vs $54.2 \%, P=.001$, respectively). As a side note, with a median follow-up time of 62 months, the median OS of the whole cohort was 101 months $(95 \%$ CI, 75.01-126.89 months), and the corresponding 5-year OS was $68 \%$. This median survival and 5-year OS contrast with the median survival of 3.5 years and 5-year OS of $36.4 \%$ from the updated PulMICC and may represent the type of selection for CRC metastasectomy to be matched with controls for inclusion in future randomized trials.

\section{RANDOMIZED TRIALS? HOW ABOUT WELL-DESIGNED, PHASE II/III PROSPECTIVE REGISTRY TRIALS FOR DISCOVERY}

So after this long diatribe suggesting that genomics may play a role in the selection of patients for pulmonary metastasectomy, one must consider that even in randomized trials that ask a specific prognostic question, how valid they will be if there are unknown or ignored prerandomization stratification issues that have an effect on the answer. Even the champions of the PulMICC trial have gone on record in their comments regarding the article on $K R A S$ and $B R A F$ mutations by Renaud and colleagues ${ }^{21}$ by confessing that "the study shows the influence of oncogenes on survival, but these are likely to be general prognostic factors, not predictors of the effectiveness of lung metastasectomy itself." ${ }^{31}$ But wait-the argument is selection. If there is a well-designed hypothesis with manageable data in both cohorts to be compared (eg, single metastasis, no liver metastases ever, never received bevacizumab), like in the Schweiger retrospective cohort, ${ }^{29}$ why couldn't one randomize to KRAS mutation or not, or even test whether Schweiger's prognostic survival and disease-free models can be validated, or at least do some preplanned post hoc analysis regarding genomic differences in the resected cohort versus the nonresected cohort?

Maybe the money is in furthering discovery, which attempts to delineate the influence of -omics in both the metastasis and the primary tumor on the efficacy of lung metastasectomy in prospective cohorts. A word of caution is in order, however. As elegantly described by Salem and 


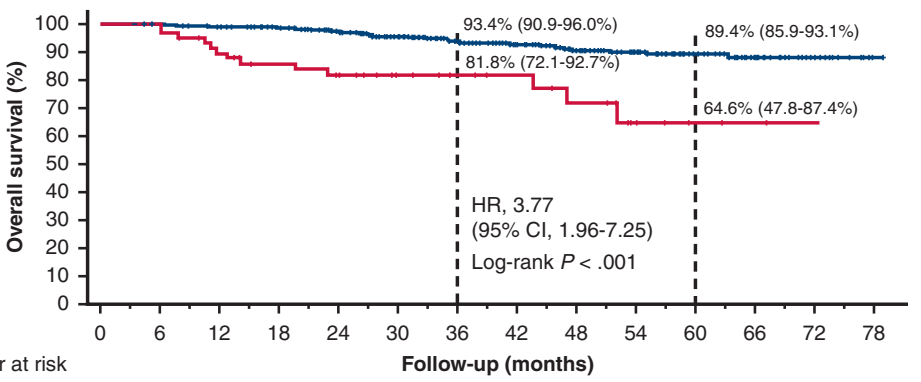

Number at risk

Post-op ctDNA status

A

\begin{tabular}{c|cccccccccccccc} 
Negative 426 & 423 & 415 & 399 & 370 & 328 & 279 & 236 & 195 & 151 & 94 & 40 & 16 & 4 \\
Positive & 59 & 59 & 50 & 44 & 37 & 27 & 22 & 17 & 12 & 7 & 4 & 3 & 2 & 1
\end{tabular}

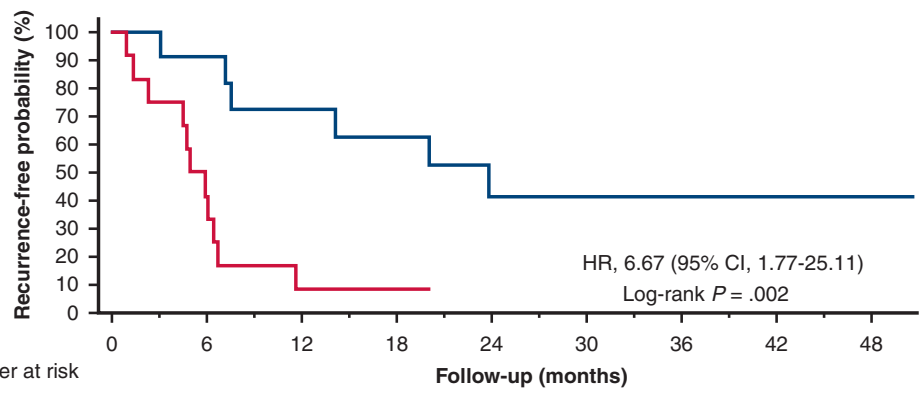

Median ctDNA MAF

\begin{tabular}{|c|c|c|c|c|c|c|c|c|}
\hline Below & 11 & 10 & 8 & 6 & 4 & 3 & 3 & 3 \\
\hline Above & 12 & 6 & 1 & 1 & 0 & 0 & 0 & 0 \\
\hline
\end{tabular}

B

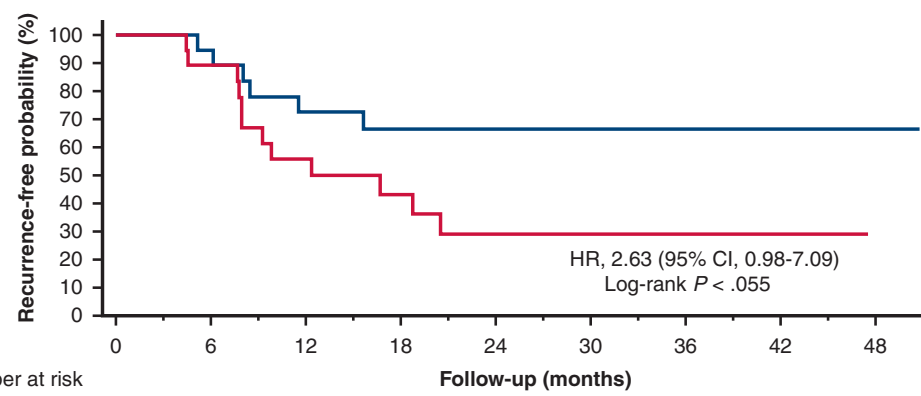

Number at risk
Median ctDNA MAF

$\simeq$\begin{tabular}{|llllllllll} 
Below & 18 & 17 & 13 & 11 & 11 & 8 & 5 & 2 & 2 \\
Above & 18 & 16 & 10 & 6 & 3 & 2 & 1 & 1 & 0
\end{tabular}

C

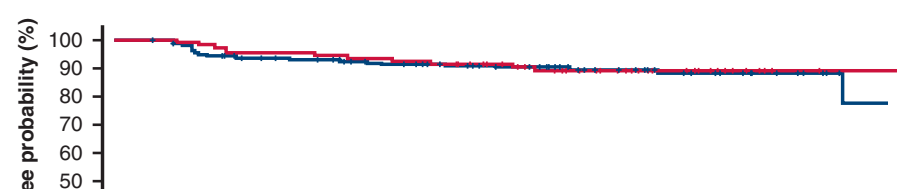

$\mathrm{HR}, 1.07$ (95\% Cl, 0.49-2.33)

Log-rank $P=.862$

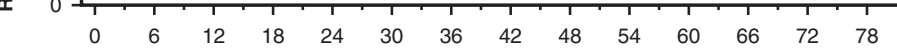

Number at risk

Follow-up (months)

Adjuvant chemotherapy

D

\begin{tabular}{|c|c|c|c|c|c|c|c|c|c|c|c|c|c|}
\hline No & 205 & 201 & 189 & 182 & 175 & 166 & 150 & 131 & 115 & 89 & 55 & 23 & 9 \\
\hline$Y \in$ & 111 & 109 & 102 & 101 & 97 & 88 & 81 & 69 & 58 & 45 & 25 & 11 & 4 \\
\hline
\end{tabular}

FIGURE 4. Circulating tumor DNA (ctDNA) and colorectal cancer (CRC) prognostication. A, CRC patients undergoing complete tumor resection have significantly longer overall survival if ctDNA is absent after resection. B-D, Mutant allele frequency (MAF), recurrence-free survival (RFS), and adjuvant chemotherapy. B, In patients not treated with chemotherapy, a higher ctDNA MAF is associated with recurrence. C, In patients treated with chemotherapy, a higher ctDNA MAF is also associated with recurrence. D, Patients with pathological node-negative disease with undetectable ctDNA after resection had equal excellent RFS irrespective of whether they received adjuvant chemotherapy. Modified from Tie and colleagues. ${ }^{34}$ 
colleagues, ${ }^{32}$ modern comparative genomic analyses of primary and metastatic colorectal tumor tissue usually demonstrates a very high concordance for driver gene mutations; however, the molecular landscape can change in response to treatment, resulting in "lesion-specific genomic heterogeneity." Thus, with multiple pulmonary metastases, a single biopsy might not provide sufficient information for prognosis and response prediction, except when there is a known single metastasis. Maybe a peripheral surrogate is needed that could correlate with the heterogeneity of the metastases and the primary. The answer may lie in Thoracic Surgery Oncology Group Trial 0905, led by Antonoff, which is already in place ${ }^{33}$ This randomized trial (Figure 3) for low-risk CRC patients with lung metastases comparing immediate resection with preoperative chemotherapy followed by resection has entry criteria similar to those of the PulMICC and will build on the fact that accrual will be higher in patients because all enrollees have a surgical option. However, the investigators are also investigating the role of changes in circulating tumor DNA (ctDNA) following surgical resection and/or systemic chemotherapy in patients with lung-limited metastatic CRC. ctDNA has already been reported to be prognostic in patients with complete resection of primary CRC. ${ }^{34}$ As shown in Figure 4, the presence of ctDNA after complete resection of CRC was associated with poorer OS, and mutant allele frequency quantification may predict those patients who experience recurrence whether or not they receive chemotherapy. Certainly in the Thoracic Surgery Oncology Group prospective trial, there is a great opportunity not only to learn whether pulmonary metastasectomy is influenced by the timing of preoperative chemotherapy in low-risk lung limited metastatic CRC, but also to define whether the presence/quantitation of ctDNA can predict which patients might not be candidates for resection in the future.

\section{WHAT IS THE TAKE HOME MESSAGE?}

Although I do not think that the PulMICC tells us anything with regard to whether pulmonary metastasectomy is efficacious, we as thoracic surgeons need to not only say that we are selective in choosing patients for metastasectomy, but also search for even more sensitive and specific tissue or blood biomarkers to justify metastasis resection. This applies not only to CRC, but also to other histologies that frequently present as oligometastatic decisions. Antonoff and colleagues lead by example in their thoughtful article and also by action in providing a model for future trials, and it is up to us to ensure that we get answers, whether we like them or not.

\section{References}

1. Antonoff MB, Sofocleous CT, Callstrom MR, Nguyen Q-N. The roles of surgery, stereotatic radiation, and ablation for treatment of pulmonary metastases. $J$ Thorac Cardiovasc Surg. 2022;163:495-502.
2. Corsini EM, Antonoff MB. Is pulmonary metastasectomy effective in prolonging survival? In: Ferguson MK, ed. Difficult Decisions in Thoracic Surgery: An Evidence-Based Approach. 4th ed. Chan, Switzerland: Springer International; 2020:279-89.

3. Schirren J, Schirren M, Lampl L, Sponholz S. Surgery for pulmonary metastases: quo vadis? Eur J Cardiothorac Surg. 2017;51:408-10.

4. Treasure T. Questioning the benefit of lung metastasectomy in colorectal cancer. BMJ. 2020;368:m264.

5. Treasure T, Farewell V, Macbeth F, Monson K, Williams NR, Brew-Graves C, et al. Pulmonary metastasectomy versus continued active monitoring in colorectal cancer (PulMiCC): a multicentre randomised clinical trial. Trials. 2019;20:718.

6. Treasure T. Metastasectomy for colorectal cancer: are there clothes on the emperor? J R Soc Med. 2017;110:227-30.

7. Milosevic M, Edwards J, Tsang D, Dunning J, Shackcloth M, Batchelor T, et al. Pulmonary metastasectomy in colorectal cancer: updated analysis of 93 randomized patients-control survival is much better than previously assumed. Colorectal Dis. 2020;22:1314-24.

8. Dolby T. She Blinded Me with Science. On: The Golden Age of Wireless. Available at: https://open.spotify.com/album/1Jsh5X2MJGGhy2S3d4b3Pb [audio file]. Accessed October 12, 2020.

9. Thibodeau SN, Bren G, Schaid D. Microsatellite instability in cancer of the proximal colon. Science. 1993;260:816-9.

10. Rajagopalan H, Bardelli A, Lengauer C, Kinzler KW, Vogelstein B, Velculescu. Tumorigenesis: RAF/RAS oncogenes and mismatch-repair status. Nature. 2002; 418:934.

11. Cancer Genome Atlas Network. Comprehensive molecular characterization of human colon and rectal cancer. Nature. 2012;487:330-7.

12. Guinney J, Dienstmann R, Wang X, de Reyniès A, Schlicker A, Soneson C, et al The consensus molecular subtypes of colorectal cancer. Nat Med. 2015;21: 1350-6.

13. Soldevilla B, Carretero-Puche C, Gomez-Lopez G, Al-Shahrour F, Riesco MC Gil-Calderon B, et al. The correlation between immune subtypes and consensus molecular subtypes in colorectal cancer identifies novel tumour microenvironment profiles, with prognostic and therapeutic implications. Eur J Cancer. 2019;123:118-29.

14. Gibbs P, Heinemann V, Sharma NK, Taieb J, Ricke J, Peeters M, et al. Effect of primary tumor side on survival outcomes in untreated patients with metastatic colorectal cancer when selective internal radiation therapy is added to chemotherapy: combined analysis of two randomized controlled studies. Clin Colorectal Cancer. 2018;17:e617-29.

15. Narayanan S, Gabriel E, Attwood K, Boland P, Nurkin S. Association of clinicopathologic and molecular markers on stage-specific survival of right versus left colon cancer. Clin Colorectal Cancer. 2018;17:e671-8.

16. Bonnot PE, Passot G. RAS mutation: site of disease and recurrence pattern in colorectal cancer. Chin Clin Oncol. 2019;8:55

17. Mukund K, Syulyukina N, Ramamoorthy S, Subramaniam S. Right- and leftsided colon cancers: specificity of molecular mechanisms in tumorigenesis and progression. BMC Cancer. 2020;20:317.

18. Lee MS, Menter DG, Kopetz S. Right versus left colon cancer biology: integrating the consensus molecular subtypes. J Natl Compr Canc Netw. 2017;15: $411-9$.

19. Schweiger T, Hegedüs B, Nikolowsky C, Hegedüs Z, Szirtes I, Mair R, et al EGFR, BRAF, and KRAS status in patients undergoing pulmonary metastasectomy from primary colorectal carcinoma: a prospective follow-up study. Ann Surg Oncol. 2014;21:946-54.

20. Renaud S, Guerrera F, Seitlinger J, Costardi L, Schaeffer M, Romain B, et al KRAS exon 2 codon 13 mutation is associated with a better prognosis than codon 12 mutation following lung metastasectomy in colorectal cancer. Oncotarget. 2017;8:2514-24

21. Renaud S, Romain B, Falcoz PE, Olland A, Santelmo N, Brigand C, et al. KRAS and BRAF mutations are prognostic biomarkers in patients undergoing lung metastasectomy of colorectal cancer. Br J Cancer. 2015;112:720-8.

22. Renaud S, Schaeffer M, Falcoz PE, Seitlinger J, Romain B, Voegeli AC, et al. Perioperative bevacizumab improves survival following lung metastasectomy for colorectal cancer in patients harbouring v-Ki-ras2 Kirsten rat sarcoma viral oncogene homologue exon 2 codon 12 mutations. Eur J Cardiothorac Surg. 2017;51:255-62.

23. National Comprehensive Cancer Network (NCCN). Colon cancer. 2020.Available at: https://www.nccn.org/professionals/physician_gls/default.aspx\#site. Accessed October 12, 2020. 
24. Corsini EM, Mitchell KG, Mehran RJ, Rice DC, Sepesi B, Walsh GL, et al. Colorectal cancer mutations are associated with survival and recurrence after pulmonary metastasectomy. J Surg Oncol. 2019;120:729-35.

25. Oudejans JJ, Slebos RJ, Zoetmulder FA, Mooi WJ, Rodenhuis S. Differential activation of ras genes by point mutation in human colon cancer with metastases to either lung or liver. Int J Cancer. 1991;49:875-9.

26. Santini D, Loupakis F, Vincenzi B, Floriani I, Stasi I, Canestrari E, et al. High concordance of KRAS status between primary colorectal tumors and related metastatic sites: implications for clinical practice. Oncologist. 2008;13:1270-5.

27. Italiano A, Hostein I, Soubeyran I, Fabas T, Benchimol D, Evrard S, et al. KRAS and BRAF mutational status in primary colorectal tumors and related metastatic sites: biological and clinical implications. Ann Surg Oncol. 2010;17:1429-34.

28. Knijn N, Mekenkamp LJ, Klomp M, Vink-Börger ME, Tol J, Teerenstra S, et al. KRAS mutation analysis: a comparison between primary tumours and matched liver metastases in 305 colorectal cancer patients. Br J Cancer. 2011;104:1020-6.

29. Schweiger T, Liebmann-Reindl S, Glueck O, Starlinger P, Laengle J, Birner P, et al. Mutational profile of colorectal cancer lung metastases and paired primary tumors by targeted next-generation sequencing: implications on clinical outcome after surgery. J Thorac Dis. 2018;10:6147-57.
30. Renaud S, Seitlinger J, Al Lawati Y, Guerrera F, Falcoz PE, Massard G, et al Anatomical resections improve survival following lung metastasectomy of colorectal cancer harboring KRAS mutations. Ann Surg. 2019;270:1170-7.

31. Cardillo G, Mokhles S, Williams N, Macbeth F, Russell C, Treasure T. Comment on: "KRAS and BRAF mutations are prognostic biomarkers in patients undergoing lung metastasectomy of colorectal cancer." Variation in survival associated with proto-oncongenes is not evidence for effectiveness of lung metastasectomy. Br J Cancer. 2015;113:1636.

32. Salem ME, Puccini A, Tie J. Redefining colorectal cancer by tumor biology. Am Soc Clin Oncol Educ Book. 2020;40:1-13.

33. American Association for Thoracic Surgery. Thoracic Surgery Oncology Group. Available at: https://www.aats.org/aatsimis/AATSWeb/Resources/Thoracic_ Surgery_Oncology_Group/. Accessed October 12,2020.

34. Tie J, Cohen JD, Lo SN, Wang Y, Li L, Christie M, et al. Prognostic significance of postsurgery circulating tumor DNA in nonmetastatic colorectal cancer: individual patient pooled analysis of three cohort studies. Int J Cancer. September 27, 2020 [Epub ahead of print].

\title{
Commentary: A final episode in the first trilogy
}

\author{
Kenneth A. Kesler, MD
}

Given that the lungs are a frequent site of hematogenous metastases from many solid neoplasms, and that continuing advances in cancer therapy are prolonging life, it can be anticipated that a growing number of patients will present with metastatic pulmonary disease. Although a multidisciplinary approach is clearly optimal to determine treatment strategies in these cases, the thoracic surgeon is not infrequently the final arbiter of not only if, but which, local therapies are used. Thus, as new technologies emerge, state-of-the-art information becomes increasingly important.

\footnotetext{
From the Division of Thoracic Surgery, Department of Surgery, Indiana University Melvin and Bren Simon Cancer Center, Indianapolis, Ind.

Disclosures: The author reported no conflicts of interest.

The Journal policy requires editors and reviewers to disclose conflicts of interest and to decline handling or reviewing manuscripts for which they may have a conflict of interest. The editors and reviewers of this article have no conflicts of interest.

Received for publication Jan 11, 2021; revisions received Jan 11, 2021; accepted for publication Jan 11, 2021; available ahead of print Jan 20, 2021.

Address for reprints: Kenneth A. Kesler, MD, Department of Surgery, Indiana University Bren Simon Cancer Center, Barnhill Dr, EM \#212, Indianapolis, IN 46202 (E-mail: kkesler@iupui.edu).

J Thorac Cardiovasc Surg 2022;163:510-1

$0022-5223 / \$ 36.00$

Copyright (c) 2021 by The American Association for Thoracic Surgery

https://doi.org/10.1016/j.jtcvs.2021.01.047
}

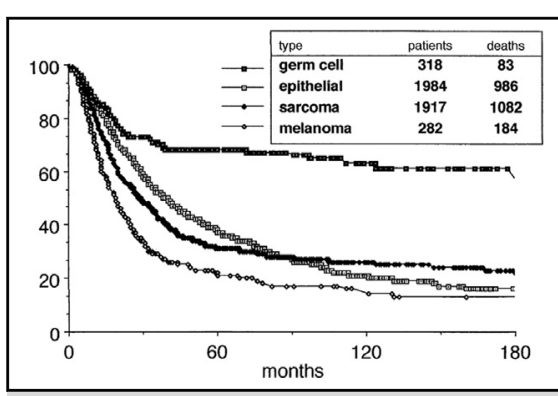

Survival after pulmonary metastasectomy according to primary tumor type. (Used with permission. ${ }^{1}$ )

\section{CENTRAL MESSAGE}

This article provides a state-ofthe-art review of local therapies for pulmonary metastatic disease, including the more recent modalities of stereotactic radiation and percutaneous ablation.

Although hundreds of retrospective, typically singleinstitution studies provide support to the safety and potential curability of surgical pulmonary metastasectomy, truly landmark publications on this topic are few and far between. Arguably pioneering publications include a report from the 